\title{
Aprendizagens realizadas pelo professor e pelos alunos: reflexão no estágio profissionalizante
}

\section{Learnings achieved by teacher and students: reflection within professional internship}

\author{
Cristina Martins, Manuel Vara Pires, João Carvalho Sousa \\ Escola Superior de Educação, Instituto Politécnico de Bragança, Portugal
}

\begin{abstract}
Resumo
Em Portugal, os mestrados profissionalizantes incluem a prática de ensino supervisionada, correspondente ao estágio de natureza profissional e objeto de relatório final, a defender em provas públicas. Na nossa instituição, este relatório deve apresentar e refletir sobre experiências de ensino e aprendizagem realizadas nos ciclos de ensino e nas disciplinas de docência. Este texto apresenta aspetos de um estudo de identificação, análise e sistematização das vertentes - conteúdo e profundidade - das reflexões escritas apresentadas na área da Matemática por doze futuros professores, no âmbito do Mestrado em ensino do $1 .^{\circ}$ e do $2 .^{\circ}$ ciclo do ensino básico.

Palavras-chave: reflexão escrita, conteúdo da reflexão, profundidade da reflexão, aprendizagens realizadas, matemática.
\end{abstract}

\begin{abstract}
In Portugal, master's degrees conducive to a professional qualification include supervised teaching practice, corresponding to in-service training, subject to a final report to be sustained in public examination. In our institution, this report must present and reflect upon teaching-learning experiences undertaken in the teaching cycles and subjects. This text presents some aspects of an identification, analysis and systematization study of the strands - content and depth - of the written reflections presented in the area of Mathematics by twelve future teachers, within the Masters for teaching in the $1^{\text {st }}$ and $2^{\text {nd }}$ cycles of basic education.

Keywords: written reflection, content of reflection, depth of reflection, learning undergone, mathematics.
\end{abstract}

\section{Apresentação e método do estudo}

Este artigo centra-se e dá continuidade ao estudo de identificação, análise e sistematização das vertentes conteúdo e profundidade — das reflexões escritas apresentadas por futuros professores nos seus relatórios finais de estágio, no âmbito do Mestrado em ensino do $1 .^{\circ}$ e do $2 .^{\circ}$ ciclo do ensino básico lecionado na nossa instituição, Escola Superior de Educação do Instituto Politécnico de Bragança, Portugal, e especificamente nas experiências de ensino e aprendizagem (EEA) desenvolvidas na área da Matemática.

Para a análise do conteúdo da reflexão seguimos três categorias: Planificação da EEA, Desenvolvimento da
EEA e Aprendizagens Efetuadas na EEA. Na categoria Planificação da EEA, foram consideradas duas subcategorias, cada uma com três indicadores: (i) Caminho percorrido: etapas seguidas na planificação, em que se baseou a planificação e seleção de objetivos, conteúdos, tarefas, recursos, gestão da sala de aula, gestão do trabalho dos alunos em sala de aula (individualmente, em pares, em grupo); e (ii) Avaliação global: importância da planificação realizada, dificuldades sentidas ao planificar e cumprimento ou não da planificação. Em Desenvolvimento da EEA, foram seguidas seis subcategorias, com os respetivos indicadores: (i) Estrutura e organização da EEA: referência às etapas da aula e sequência da aula; (ii) Organização e gestão da sala de aula: contexto da turma, organização do tempo, organização do espaço e organização do trabalho em sala de aula (individual, em pares, em grupo); (iii) Comunicação na sala de aula: questões surgidas, debates e discussão e partilha de ideias; (iv) Atividade do aluno - tarefas: referência ao enunciado e à resolução das tarefas, papel dos alunos nos vários momentos da EEA, estratégias de resolução utilizadas, produções dos alunos e utilização e exploração de recursos materiais; (v) Atividade do aluno - atitudes: atitudes, envolvimento, modo de estar na sala de aula e dificuldades em relação ao processo; e (vi) Atividade do professor: papel do professor nos vários momentos da aula, atitudes, envolvimento, modo de estar na sala de aula e dificuldades em relação ao processo. Finalmente, na categoria Aprendizagens Efetuadas na $E E A$, foram consideradas duas subcategorias, cada uma com três indicadores: (i) Aprendizagens dos alunos: o que os alunos terão aprendido sobre a Matemática, dificuldades sentidas e fatores que contribuíram ou dificultaram a aprendizagem; e (ii) Aprendizagens do professor: o que aprendeu o professor com esta EEA, dificuldades sentidas e fatores que contribuíram ou dificultaram a aprendizagem. Para a análise da profundidade seguimos três categorias a priori, baseadas nos níveis definidos por Lee (2005): (1) nível de recordação: verificado quando o futuro professor descreve o que experiencia, e interpreta a situação recordando as suas experiências, sem considerar explicações alternativas; (2) nível de racionalização: 
verificado quando o futuro professor procura relações entre partes das suas experiências, interpreta a situação racionalmente, procura justificações para os acontecimentos, e generaliza as suas experiências ou produtos com princípios orientadores; e (3) nível de reflexividade: verificado quando o futuro professor aborda as suas experiências com a intenção de mudar ou melhorar no futuro, analisa as suas experiências a partir de várias perspetivas, e é capaz de ver a influência dos professores orientadores nos seus valores, comportamento e realizações.

Do trabalho desenvolvido, destacamos que, da primeira etapa, correspondente à análise do conteúdo das reflexões escritas, decorreu uma maior a incidência da reflexão na categoria Desenvolvimento da EEA (sendo as outras categorias Planificação da EEA e Aprendizagens Efetuadas na EEA). Da segunda etapa, centrada na análise da profundidade alcançada nas reflexões, ficou evidenciada a presença de todos os níveis de reflexão (nível de recordação; nível de racionalização; e nível de reflexividade), sendo, porém, percetível alguma variação conforme a categoria ou subcategoria em que incide a reflexão. A maior percentagem de cada nível corresponde a uma categoria distinta. Da terceira etapa, verificámos a articulação manifestada entre as vertentes em estudo. Concluímos que, na categoria Planificação da EEA, verificou-se que os indicadores que manifestam maior percentagem a nível da incidência do conteúdo se dispersam pelos diferentes níveis de profundidade. $\mathrm{Na}$ categoria Desenvolvimento da EEA, os indicadores com maior expressividade na vertente da reflexão concentram-se, sobretudo, em dois níveis de profundidade, verificando-se indicadores com percentagem nula. $\mathrm{Na}$ categoria Aprendizagens Efetuadas na EEA, os futuros professores refletiram sobre todos os indicadores, sendo nesta categoria que se destaca o nível de reflexividade, especificamente, com uma percentagem muito significativa na subcategoria Aprendizagens do professor.

Tendo em consideração este facto e à semelhança de um trabalho anterior, subsequente à última etapa acima identificada, incidente na categoria Desenvolvimento da EEA (por ser aquela em que prevalece o nível de recordação), considerámos pertinente avançar com uma análise particular, alvo do presente artigo, e operacionalizada na seguinte questão: $\mathrm{Na}$ categoria Aprendizagens realizadas na EEA, afinal, sobre que aspetos refletem os futuros professores e com que profundidade?

Em termos metodológicos, o objetivo da análise nesta fase foi fundamentalmente de transcender a análise realizada (análise de conteúdo transversal de um corpus constituído pela totalidade de doze relatórios finais de estágio) e determinar o tipo e forma das representações dos sujeitos em relação aos itens definidos na fase de categorização (Carley \& Palmquist, 1992). Foram utilizados recursos informáticos adequados, especificamente NVivo (QSR International Pty Ltd., 2012), para fazer o levantamento e tratamento estatístico da informação recolhida e todos os segmentos encontrados foram codificados em função das duas vertentes da reflexão — conteúdo e profundidade.
Procedeu-se em seguida a uma análise de tipo relacional, categorizando cada segmento previamente codificado em função do indicador utilizado para essa categorização, e, dentro de cada indicador, a expressão alcançada por cada um dos níveis de reflexão considerados.

\section{Aprendizagens realizadas na EEA: conteúdo e profundidade presente nas reflexões dos futuros professores}

Para uma melhor organização deste ponto, referimos dois subpontos: um dedicado ao conteúdo e outro à profundidade, incluindo neste último a articulação com o anterior.

\section{Conteúdo da reflexão: subcategorias e indicadores em destaque}

Neste subponto é nossa intenção dar resposta à questão: Em que subcategorias e respetivos indicadores recai, com maior expressão, a reflexão na categoria Aprendizagens realizadas na EEA?

$\mathrm{Na}$ Tabela 1, apresentamos a incidência da reflexão nos indicadores das referidas subcategorias.

Tabela 1.

Incidência da reflexão na categoria "Aprendizagens realizadas na EEA",

\begin{tabular}{llc}
\hline Subcategorias & Indicadores & $\begin{array}{c}\text { Incidência } \\
\text { do conteúdo }\end{array}$ \\
\hline $\begin{array}{l}\text { Aprendizagens } \\
\text { dos alunos }\end{array}$ & $\begin{array}{l}\text { - o que os alunos terão } \\
\text { aprendido sobre a Matemática }\end{array}$ & $59,57 \%$ \\
& - dificuldades sentidas & $23,40 \%$ \\
& - fatores que contribuíram ou & $17,03 \%$ \\
& dificultaram a aprendizagem & \\
\hline Aprendizagens & - o que aprendeu o professor & $44,00 \%$ \\
do professor & com esta EEA & \\
& - dificuldades sentidas & $4,00 \%$ \\
& - fatores que contribuíram ou & $52,00 \%$ \\
& dificultaram a aprendizagem & \\
\hline
\end{tabular}

Pela análise da tabela verifica-se que os indicadores "o que os alunos terão aprendido sobre a Matemática", na subcategoria Aprendizagens dos alunos, e "fatores que contribuíram ou dificultaram a aprendizagem", na subcategoria Aprendizagens do professor, recolheram uma maior incidência, com uma percentagem superior a $50 \%$. Também o indicador "o que aprendeu o professor com esta EEA", da subcategoria Aprendizagens do professor, foi bastente referido, apresentando $44 \%$ de incidência. Os restantes manifestam percentagens bastante inferiores.

\section{Profundidade da reflexão: subcategorias e indicadores em destaque}

$\mathrm{Na}$ análise anteriormente mencionada, no respeitante à profundidade da reflexão, foi visível que, nas três categorias consideradas, o nível de reflexividade surge em maior percentagem em Aprendizagens realizadas na $E E A$, embora, nesta categoria, este nível seja o intermédio, dado que o nível de recordação atinge a maior percentagem e o nível de racionalização a menor. Particularmente, o nível de reflexividade surge com uma 
percentagem muito significativa, acima de $90 \%$, na subcategoria Aprendizagens do professor.

Ora, apesar de o nível de reflexividade ser o mais expressivo nesta subcategoria, em que indicadores se centra? E na outra subcategoria o que se verifica?

Para dar resposta a estas questões apresentamos, na Tabela 2, a percentagem da incidência da profundidade nos três níveis de reflexão adotados.

Tabela 2.

Incidência da profundidade da reflexão na categoria "Aprendizagens realizadas na EEA"

\begin{tabular}{llccc}
\hline \multirow{2}{*}{$\begin{array}{l}\text { Sub- } \\
\text { categorias }\end{array}$} & Indicadores & \multicolumn{3}{c}{$\begin{array}{c}\text { Incidência da } \\
\text { profundidade }\end{array}$} \\
\cline { 3 - 5 } & & $\mathrm{N} 1$ & $\mathrm{~N} 2$ & $\mathrm{~N} 3$ \\
\hline $\begin{array}{l}\text { Aprendiza- } \\
\text { gens dos }\end{array}$ & - o que os alunos terão & 75,00 & 10,71 & 14,29 \\
alunos & Matemdido sobre a & $\%$ & $\%$ & $\%$ \\
& - dificuldica & & & \\
& & 90,91 & 9,09 & 0,00 \\
& - fatores que contribuí- & 12,50 & 62,50 & $\begin{array}{c}25,00 \\
\%\end{array}$ \\
& ram ou dificultaram a & $\%$ & $\%$ & $\%$ \\
& aprendizagem & & & \\
\hline Aprendiza- & - o que aprendeu o & 0,00 & 27,27 & 72,73 \\
gens do & professor com esta EEA & $\%$ & $\%$ & $\%$ \\
professor & - dificuldades sentidas & 0,00 & 0,00 & 100,0 \\
& & $\%$ & $\%$ & $\%$ \\
& - fatores que contribuí- & 0,00 & 30,77 & 69,23 \\
& ram ou dificultaram a & $\%$ & $\%$ & $\%$ \\
& aprendizagem & & & \\
\hline
\end{tabular}

Conforme é notório na Tabela 2, na subcategoria Aprendizagens do professor, o nível de reflexividade foi, de facto, evidente, atingindo em todos os indicadores percentagens superiores a $69 \%$. Concretamente, no indicador em que se verifica maior percentagem de incidência do conteúdo, "fatores que contribuíram ou dificultaram a aprendizagem", a profundidade centra-se nos níveis de reflexividade $(69,23 \%)$ e racionalização $(30,77 \%)$, originando uma percentagem nula no nível de recordação. No indicador "o que aprendeu o professor com esta EEA", em que a percentagem de incidência do conteúdo é de $44 \%$, a profundidade centra-se também nos níveis de racionalização e reflexividade e, igualmente, com grande expressividade no nível de reflexividade $(72,73 \%)$. O indicador "dificuldades sentidas", embora com uma pequena incidência no conteúdo, focaliza-se unicamente no nível de reflexividade.

Relativamente ao indicador "o que os alunos terão aprendido sobre a Matemática", da subcategoria Aprendizagens dos alunos, cuja incidência do conteúdo foi a mais significativa $(59,57 \%)$, a reflexão focaliza-se nos três níveis, revelando, contudo, uma incidência muito significativa da profundidade no nível de recordação, com $75,00 \%$, seguindo os restantes níveis com percentagens pouco relevantes: nível de reflexividade - $14,29 \%$ e nível de racionalização $10,71 \%$.

Em relação aos restantes indicadores desta subcategoria, "dificuldades sentidas" e "fatores que contribuíram ou dificultaram a aprendizagem", com percentagens mais baixas de incidência na componente do conteúdo, respetivamente $23,40 \%$ e $17,02 \%$ verifica-se que o primeiro se centra fortemente no nível de recordação $(90,91 \%)$ e apenas $9,09 \%$ no nível de racionalização e o segundo centra-se nos três níveis, com maior expressividade no nível de racionalização $(62,50 \%)$ e menos nos outros (reflexividade $-25,00 \%$ e recordação - 12,50\%).

\section{Nível de reflexividade: evidências e recomendações}

Para terminar, e dando continuidade ao objetivo deste trabalho, consideramos essencial dar resposta às questões: Quais as evidências que comprovam a incidência no nível de reflexividade? De que forma se interligam com as ideias defendidas na literatura?

No indicador "o que aprendeu o professor com esta EEA" foi destacado o reconhecimento da importância e das potencialidades das tarefas matemáticas realizadas, $\mathrm{o}$ que pode ser constatado, por exemplo, na evidência " $\mathrm{E}$ reconhecemos que o trabalho realizado foi enriquecedor e que este tipo de tarefas promoveu possibilidades de compreensão e de sucesso aos alunos, especialmente àqueles com mais dificuldades". A respeito dos diferentes tipos de tarefas que os professores podem desenvolver nas suas aulas, Stein, Engle, Smith e Hughes (2008) vincam que quando estes realizam tarefas de ensino centradas no aluno enfrentam desafios que devem ir além da apropriada identificação de tarefas e da sua aplicação adequada na sala de aula. Dado que nestas tarefas normalmente os caminhos para chegar à solução não são especificados, os alunos tendem a realizá-las de forma única e, por vezes, inesperada. Os professores não devem apenas esforçar-se para entender a forma como os alunos estão a interpretar a tarefa, mas também começar a categorizar as diferentes ideias e abordagens dos alunos de acordo com a natureza da matemática. De facto, só formulando tarefas adequadas é que o professor pode suscitar a atividade do aluno (Ponte, 2005). Como referem Martins, Maia, Menino, Rocha e Pires (2002), é importante que os alunos, quando trabalham conceitos matemáticos, sejam confrontados com diferentes tipos de tarefas, quer sejam exercícios mais orientados para aspetos rotineiros, quer sejam problemas ou investigações apelando mais ao trabalho exploratório e não rotineiro. E adiantam que uma boa tarefa pode ser completamente desaproveitada por uma deficiente exploração ou, inversamente, uma tarefa do tipo exercício pode, através de uma orientação ou exploração adequadas, conduzir a um trabalho significativo. Num sentido semelhante, Ponte (2005) alerta para o facto de não ser suficiente "selecionar boas tarefas - é preciso ter atenção ao modo de as propor e de conduzir a sua realização na sala de aula" (p. 12). A este respeito, foi também possível verificar que houve registos de futuros professores que destacaram a relevância do tempo, e da sua gestão, que é necessário disponibilizar na realização das tarefas: "Foi importante dar tempo para que os alunos pudessem pensar e resolver as questões, sendo uma mais-valia para que eles chegassem à resposta". 
No indicador "dificuldades sentidas", manifestou-se incidência exclusiva na organização do trabalho dos alunos em sala de aula, como se pode verificar na evidência "[A gestão dos grupos não foi fácil] pensamos que a tarefa teria sido mais produtiva se os grupos fossem similares". A este respeito, no trabalho desenvolvido por Baía (2013), os participantes no estudo apresentaram diferentes perspetivas e práticas. Por exemplo, Sandra valorizava o trabalho individual, realizando, sobretudo, trabalho em pares por considerar ser mais prático em termos de gestão do espaço e otimização do tempo. Miguel considerava a organização de grupos heterogéneos, com alunos de diferentes níveis de compreensão, onde uns pudessem aprender com a ajuda dos outros. Por isso, Ponte et al. (2007) referem que a "aprendizagem da Matemática pressupõe que os alunos trabalhem de diferentes formas na sala de aula" (p. 10). Relativamente a questões de natureza organizativa da atividade dos alunos, e para que trabalho de grupo funcione plenamente, Abrantes, Leal, Teixeira e Veloso (1997) consideram que o professor, embora deva ser flexível quanto à composição e estabilidade dos grupos e atender às preferências dos alunos, deve assumir o papel de orientar a formação dos grupos, nomeadamente quando já conhece melhor os alunos, bem como propor alterações quando considerar que é conveniente. Especificamente, sobre a organização do trabalho em díade, César (2000) destaca que esta forma de organização promove o desenvolvimento cognitivo dos alunos e os seus desempenhos matemáticos, assinalando que, desta forma, os alunos têm de ser capazes de recontextualizar o que sabem para poderem estabelecer uma intersubjectividade comum com o seu par. Assim, têm de conseguir descentrar-se das suas posições, para poderem compreender estratégias de resposta diferentes, para conseguirem seguir os raciocínios um do outro. Para a criação de uma cultura de trabalho de grupo na aula de Matemática, Ponte et al. (2007) reforçam que é necessário "sensibilizar os alunos para a importância da definição de objetivos comuns, a estruturação e calendarização do trabalho, tomada de iniciativas e assunção de responsabilidades, procurando desenvolver neles tanto a sua autonomia como o espírito de colaboração" (p. 10).

No indicador "fatores que contribuíram ou dificultaram a aprendizagem" sobressaiu, sobretudo, o reconhecimento da importância da reflexão sobre a prática, como é notória na seguinte evidência: "Terá sido o raciocínio matemático desenvolvido ao longo destes últimos cinco anos de escolaridade? Terá existido a preocupação de serem planeadas algumas atividades que os levam a interrogarem-se?. Sem respostas, a certeza foi e é apenas uma, são obrigatórias em qualquer área disciplinar atividades que promovam a discussão, o raciocínio e a comunicação". A reflexão apresenta-se como o elo de ligação entre o conhecimento e a prática (Krainer, 1996; Menezes \& Ponte, 2006). É uma das atividades mais apontadas para contribuir para o desenvolvimento profissional do professor, uma vez que se pode apresentar como um meio propício para enfrentar situações novas e para melhorar as práticas de sala de aula (Martins, 2011, Menezes \& Ponte, 2006; Schön, 1983; Zeichner, 1993).

De facto, como afirmam Stein e Smith, (2009), "embora todos os professores pensem informalmente acerca das suas experiências de sala de aula, cultivar hábitos de reflexão ponderada e sistemática pode ser a chave tanto para melhorar o seu ensino como para sustentar o seu desenvolvimento profissional ao longo da vida" (p. 22). A concluir, e para a promoção da formação e desenvolvimento profissionais do professor, consideramos fundamental considerar, entre outros, os seguintes aspetos: (i) a importância da reflexão dos professores sobre as suas práticas e experiências de aprendizagem (Day, 2001; Martins \& Pires, 2008; Serrazina, 1998; Zaslavsky, Chapman \& Leikin, 2003); (ii) a valorização da compreensão da Matemática que se vai ensinar (Borko, 1994; Conference Board of the Mathematics Sciences, 2001; Ma, 1999; National Council of Teachers of Mathematics, 2017; Sowder, 2007; Zaslavsky, Chapman \& Leikin, 2003; Wu, 1999); (iii) a relevância do papel do supervisor ou formador ou acompanhante ou amigo crítico ou agente de mudança (Day, 2001; Putman, Smith \& Cassady 2009; Sachs, 2009; Serrazina, Canavarro, Guerreiro, Rocha, Portela \& Saramago, 2005); e (iv) o reconhecimento do papel ativo do professor no seu próprio desenvolvimento profissional (Guimarães, 2006; Rocha \& Fiorentini, 2006).

\section{Referências}

Abrantes, P., Leal, L., Teixeira, P. \& Veloso, E. (1997). $M_{789}$. Inovação curricular em matemática. Lisboa: Fundação Calouste Gulbenkian.

Baía, I. (2013). Perceções dos professores de matemática do ensino básico acerca do trabalho de grupo na sala de aula. Dissertação de Mestrado, Instituto Politécnico de Bragança, Bragança, Portugal.

Borko, H. (2004). Professional development and teacher learning: mapping the terrain. Educational Researcher, 33(8), 3-15.

Carley, K. \& Palmquist, M. (1992). Extracting, representing and analyzing mental models. Social Forces, 70(3), 601-636.

César, M. (2000). Interações sociais e apreensão de conhecimentos matemáticos: a investigação contextualizada. In J. P. Ponte \& L. Serrazina (Eds.), Educação matemática em Portugal, Espanha e Itália: Atas da Escola de Verão 1999 (pp. 5-46). Lisboa: Secção de Educação e Matemática da Sociedade Portuguesa de Ciências da Educação.

Conference Board of the Mathematics Sciences. (2001). The mathematical education of teachers (Issues in Mathematics Education, Vol. 11). Washington: Mathematical Association of America \& American Mathematical Society.

Day, C (2001). Desenvolvimento profissional de professores: os desafios da aprendizagem permanente. Porto: Porto Editora.

Guimarães, F. (2006). Como se pensa hoje o desenvolvimento profissional?. Quadrante, 15, 169-192. 
Krainer, K. (1996). In-service education as a contribution to the improvement of professional practice: some insights into an Austrian in-service programme for mathematics teacher. In J.P. Ponte, C. Monteiro, M. Maia, L. Serrazina \& C. Loureiro (Eds.), Desenvolvimento profissional de professores de matemática: que formação? (pp. 155-171). Lisboa: Secção de Educação e Matemática da Sociedade Portuguesa de Ciências da Educação.

Lee, H. (2005). Understanding and assessing preservice teachers' reflective thinking. Teaching and Teacher Education, 21, 699-715.

Ma, L. (2009). Saber e ensinar matemática elementar. Lisboa: Gradiva.

Martins, C. (2011). O desenvolvimento profissional de professores do $1 .^{\circ}$ ciclo: contributo da participação num programa de formação contínua em matemática. Tese de Doutoramento, Universidade de Lisboa, Lisboa, Portugal.

Martins, C., Maia, E., Menino, H., Rocha, I. \& Pires, M.V. (2002). O trabalho investigativo nas aprendizagens iniciais da matemática. In J.P. Ponte et al. (Orgs.), Atividades de investigação na aprendizagem da matemática e na formação de professores (pp. 59-81). Coimbra: Secção de Educação e Matemática da Sociedade Portuguesa de Ciências da Educação.

Martins, C. \& Pires, M.V. (2008). O que é uma boa reflexão sobre a prática?. Educação e Matemática, 98, 24-27.

Menezes, L. \& Ponte J.P. (2006). Da reflexão à investigação: percursos de desenvolvimento profissional de professores do $1 .^{\circ}$ ciclo na aula de matemática. Quadrante, 15, 145-168.

National Council of Teachers of Mathematics. (2017). Princípios para a ação: assegurar a todos o sucesso em matemática. Lisboa: Associação de Professores de Matemática.

Ponte, J.P. (2005). Gestão curricular em matemática. In GTI (Ed.), O professor e o desenvolvimento curricular (pp. 11-34). Lisboa: Associação de Professores de Matemática.

Ponte, J.P., Serrazina, L., Guimarães, H., Breda, A., Guimarães, F., Sousa, H., Menezes, L., Martins, M. G. \& Oliveira, P. (2007). Programa de matemática do ensino básico. Lisboa: Ministério da Educação.

Putman, M.S., Smith, L.L. \& Cassady, J.C. (2009). Promoting change through professional development: the place of teacher intentionality in reading instruction. Literacy Research and Instruction, 48(3), 207-220.

Rocha, L. \& Fiorentini, D. (2006). Desenvolvimento profissional de professores de matemática em início de carreira no Brasil. Quadrante, 15, 145-169.

Sachs, J. (2009) Aprender para melhorar a aprendizagem: o dilema do desenvolvimento profissional contínuo dos professores. In M. Flores \& A. Veiga Simão (Orgs.), Aprendizagem $e$ desenvolvimento profissional de professores: contextos $e$ perspetivas (pp. 99-118). Mangualde: Edições Pedago.
Schön, D. (1983). The reflective practitioner: how professionals think in action. Aldershot Hants: Avebury.

Serrazina, L. (1998). Teacher's professional development in a period of radical change in primary mathematics education in Portugal (Tese de Doutoramento, Universidade de Londres, UK). Lisboa: Associação de Professores de Matemática.

Serrazina, L., Canavarro, A., Guerreiro, A., Rocha, I., Portela, J. \& Saramago, M.J. (2005). Programa de formação contínua em matemática para professores do 1. ${ }^{\circ}$ ciclo. Lisboa: Ministério da Educação.

Sowder, J.T. (2007). The mathematical education and development of teachers. In F. Lester (Ed.), Second handbook of research on mathematics teaching and learning: a project of the National Council of Teachers of Mathematics (pp. 157-223). Charlotte: Information Age Publishing.

Stein, M.K., Engle, R., Smith, M. \& Hughes, E. (2008) Orchestrating productive mathematical discussions: five practices for helping teachers move beyond show and tell. Mathematical Thinking and Learning, 10(4), 313-340.

Stein, M.K. \& Smith, M.S. (2009). Mathematical tasks as a framework for reflection: from research to practice. Educação e Matemática, 105, 22-28.

$\mathrm{Wu}, \quad$ H. (1999). Professional development of mathematics teachers. American Mathematical Society, 46(5), 535-542.

Zaslavsky, O., Chapman, O. \& Leikin, R. (2003). Professional development of mathematics educators: trends and tasks. In A.J. Bishop, M.A. Clements, C. Keitel, J. Kilpatrick \& F.K. Leung (Eds.), Second international handbook of mathematics education part two. (pp. 877-917). Dordrecht, The Netherlands: Kluwer Academic Publishers.

Zeichner, K. (1993). A formação reflexiva de professores: ideias e práticas. Lisboa: Educa. 